\title{
Is the degree of demoralization found among refugee and migrant populations a social-political problem or a psychological one?
}

\author{
Lynne Briggs
}

School of Human Services and Social

Work, Griffith University, Southport

AUSTRALIA

\begin{abstract}
Background and Objectives: Many international studies point to the negative impact of migration on refugee mental health while others consider the social and political aspects of resettlement are more important. This paper presents the findings from studies examining the degree of demoralization and the impact of other factors on resettlement among three cohorts of resettled refugees and migrant people residing in Australia, Canada and New Zealand.

The aims were to determine: participant levels of demoralization; ascertain if the goals contained in the New Zealand Immigration Settlement Strategy are achievable and whether the lack of such goals impacted on participant levels of demoralization.

Methods: Study questionnaires, standardized inventories, focus groups, individual semistructured interviews, and a demoralisation scale were completed by three different cohorts of refugee and migrant people attending mental health and resettlement services in Australia, Canada and New Zealand. The data was analyzed using statistical and thematic analysis.

Results: While a degree of demoralization was evident across all cohorts significant differences $(\mathrm{p}<0.01)$ were found between mental health participant scores in comparison to non-clinical cohorts. Factors such as an ability to speak English $(\mathrm{p}<0.01)$ and unemployment $(\mathrm{p}<0.001)$ also significantly impacted on the demoralization mean scores.

Conclusions: The findings support the view that social and cultural issues play a role in understanding the degree of psychological distress among culturally diverse clients. Thus, in order to reduce the risk, additional factors associated with migration that may impact on resettlement need to be taken into account.
\end{abstract}

Received: 1 October 2012

Revised: 30 November 2012

Accepted: 19 December 2012

The three studies were funded by the University of Otago, New Zealand. 


\section{Introduction}

Refugee migration generally means resettlement in a country of a very different culture. This takes considerable adjustment as ties with family and friends are severed ${ }^{1-4}$, individuals with higher levels of education in their home countries may experience a decrease in socioeconomic status in resettlement or become unemployed $^{5-10}$. It also takes time to acquire the host country's language and to understand the cultural milieu of that country ${ }^{1}$. A combination of these factors alongside the host society's socioeconomic-political situation can impact negatively on an individual's mental health and wellbeing ${ }^{1,2,4,5}$.

Furthermore, as several researchers ${ }^{1-4}$ have noted, refugees and migrant people coming from non-English speaking cultures have either no way of understanding the concept of mental ill health, or they associate it with the more severe disorders that require psychiatric intervention. This adds to the considerable adjustment already required by refugees and their host societies.

New Zealand has been offering third country resettlement to refugees and their families since the end of the Second World War. While many studies in the international literature point to the negative impact of migration on the mental health of individuals, others consider whether the social and political aspects of resettlement are more important as adjustment begins during the post-migration period where the realities of migration become more apparent ${ }^{1-4}$. That is, the combination of being faced with the realities of migration alongside social isolation from extended family and friends can result in individuals feeling a sense of alienation and failure, which in turn leads to existential distress, depression and/or, demoralization ${ }^{1,4}$.

Demoralization has been described as a change in morale spanning a spectrum of mental attitudes from disheartenment (mild loss of confidence) through despondency (starting to give up) and to actually having 'given up ${ }^{1,11,12}$. Also noted ${ }^{13}$, is the debate surrounding the concept of demoralization with some questioning its value, while others claim its usefulness in explaining non-specific psychological distress. The main controversy centres on whether feelings of demoralization constitute a syndrome of despair, distress and hopelessness separate from depression, or reflect clinical depression, or are a normal response to difficult circumstances. It has been $\operatorname{argued}^{11-13}$ that the critical feature distinguishing depression from demoralization is the presence or absence of anhedonia (a diminished ability to experience pleasure). That is, a depressed person has lost the ability to experience pleasure generally, as well as motivation and drive, even when an appropriate direction of action is known.

In contrast to depression, a feeling of subjective incompetence and helplessness ${ }^{13}$ characterizes demoralization. A demoralized person, while unable to look forward with pleasant anticipation, may laugh and enjoy the present moment, but feels helpless, incompetent and inhibited in action by not knowing what to do $\mathrm{d}^{11-13}$ a feature noted by the researchers when working clinically with refugees ${ }^{1,4}$. Thus, the concept of demoralization is of particular interest to professionals offering services to refugee and migrant clients as it contains a lot of negative symptoms and feelings that may be experienced as a result of resettlement, particularly if this has occurred through forced migration. Such symptoms are intensified if the host country is of a different culture ${ }^{1-4}$.

This paper briefly presents the findings from a programme of research undertaken over several years examining the degree of demoralization among three cohorts of resettled refugees and migrant people coming from 
refugee like backgrounds residing in Australia, Canada and New Zealand. The key findings from the studies are used as an illustration of the impact that other factors known to influence successful resettlement may have on an individual's demoralization score.

\section{The Studies}

\section{Study One}

The first study, undertaken between 2005 and 2008, involved 50 clients attending a mental health service (MHS) and 25 attending a resettlement service in New Zealand (CRS) with another 25 attending community services dealing with resettlement issues in South Australia (AUR) ${ }^{1}$. The aim was to determine the degree of demoralization among refugee populations presenting at these services and whether demoralization may provide a more relevant diagnosis than depression for refugee and migrant clients with mental health problems. Ascertaining if other factors known to assist successful resettlement also impacted on the participants' demoralization scores was also explored.

\section{Methods}

A full description of the methodology used, analysis of the data and findings for this study have been reported elsewhere ${ }^{1}$. In brief, it included the use of a Demoralization Scale (DS). This 24-item instrument captures the dimensions of demoralization in its subscales: dysphoria; disheartenment; loss of meaning; helplessness; and sense of failure ${ }^{14}$ and correlates well with Beck's BDI-II and BHS scales ${ }^{15,16}$, thus promising good psychometric properties. It was also found to have divergent validity, demonstrated through the differentiation of a sub-group of patients with high demoralization who did not meet American Psychiatric Association's 'Diagnostic and Statistical Manual of Mental Disorders (DSM-IV) ${ }^{17}$, criteria for a diagnosis of major depressive disorder.

To ensure the DS was suitable for use with this non-Western sample the instrument required modification in the way the questions were asked, including finding proper and consistent substitutes for words, symptoms and states of mind, which are part of linguistic and cultural idiosyncrasies. Thus, interpreters trained in working with people from the different cultures were engaged so that all participants were able to give informed consent and understand the questions being asked.

Whilst scoring for the DS can range between 0-96, rising scores indicate an increased level of severity of the phenomena experienced. Beck's BDI-II and BHS scales ${ }^{16,17}$, were also used in the study reported here ${ }^{1}$. As Pearson correlations demonstrated, significant associations between the three measures used ( $p<0.001)$, similar cut-off points for the DS (<34 mild, 35-59 moderate and >60 severe) were established to allow for the level of severity for demoralization among the sample to be estimated.

\section{Results}

As shown in Table 1 below, the mean total sample score on the DS (mean $=39.51$; SD $=19.48$ ) indicated that many of participants in the total sample were experiencing a moderate level of demoralization. Also shown is that while the resettlement services' total DS mean scores $(\mathrm{CRS}=32.76$ : SD: 18.53; AUR $=29.76$ : SD: 17.05), were lower than that of the Mental Health Service's total mean score (MHS = 47.76: SD 17.74) it was interesting to note that these scores were also trending toward a moderate level of demoralization. 


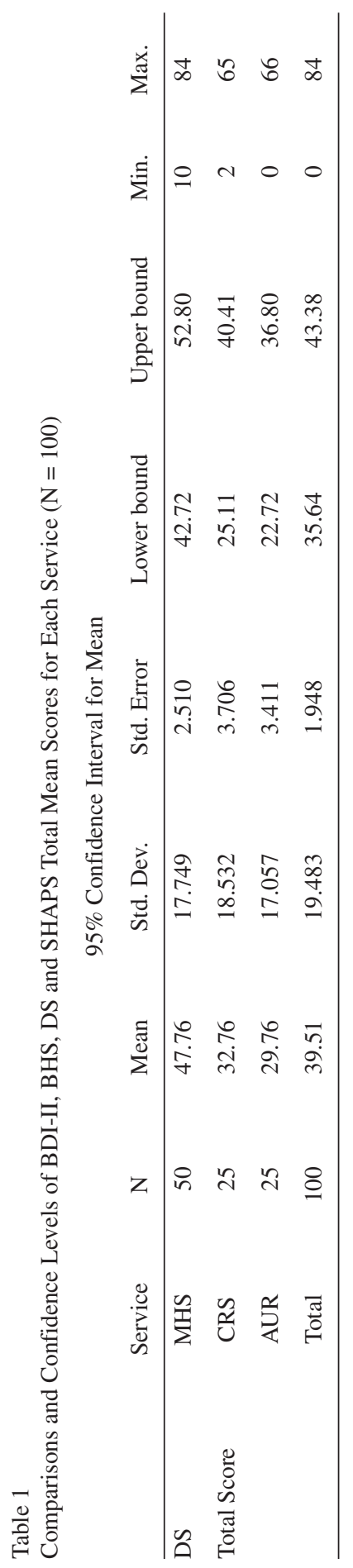

Using the same clinical cut-off points again, a further analysis was undertaken in an attempt to ascertain any significant differences between depression with anhedonia and depression with demoralization. Although the findings did not allow for determination as to whether demoralisation could be seen as a more relevant diagnosis than major depressive disorder, the overall results did indicate that DS may be an applicable measure of non-specific distress that spans a spectrum from mild disheartenment through to total despondency.

Further investigation of other clinical factors found that demoralization was significantly associated with variables such as somatic complaints (mean $=44.65 ; \mathrm{SD}=17.74$, $\mathrm{p}<0.001$ ), diagnosis (mood/anxiety disorder mean $=48.89 ; \mathrm{SD}=17.01 ;$ no mood/anxiety disorder mean $=27.60 ; \mathrm{SD}=15.94, \mathrm{p}<0.001$ ), antidepressant intervention (medicated mean $=48.72 ; \mathrm{SD}=17.09 ;$ not medicated mean $=$ 31.34; $\mathrm{SD}=17.87, \mathrm{p}<0.001)$ and response to antidepressants (effective mean $=36.40$; $\mathrm{SD}=15.44$, not effective mean $=52.05, \mathrm{SD}$ $=16.12, \mathrm{p}<0.001)^{1}$.

While other social factors in literature cited to be significant in resettlement, such as having family in NZ, proficiency in English and time in the country were explored, no significant associations were found. The exception, to this, which is of importance to this paper, was finding a highly significant association ( $\mathrm{p}$ $<0.001$ ) between employment and participant demoralization scores and unemployment and participant demoralization scores (employed mean $=33.18 ; \mathrm{SD}=20.50$ : unemployed mean $=43.73 ; \mathrm{SD}=17.71)^{1}$, thus demonstrating that social issues such as unemployment can impact seriously on the mental health status of an individual. 


\section{Studies Two and Three}

The aim of the second and third studies undertaken in Canada during 2008-2009, and repeated in New Zealand between 2010-2011, was to determine if the goals contained in the New Zealand Immigration Settlement Strategy $(\mathrm{NZSS})^{18}$ were achievable and to ascertain if a lack of achievement of the goals impacted on participant levels of demoralization.

The goals contained in the NZSS include the following:

1. Obtain employment appropriate to qualifications and skills;

2. Are confident in using English in a New Zealand setting or;

3. Can access appropriate language support to bridge the gap;

4. Access appropriate information and responsive services that are available to the wider community (for example housing, education, and services for children);

5. Form supportive social networks and establish a sustainable community identity;

6. Feel safe expressing their ethnic identity and are accepted by, and are part of, the wider host community; and participate in civic, community and social activities;

7. Accept and respect the New Zealand way of life and contribute to civic, community and social activities.

It was thought that given Canada's reputation for successful resettlement and for addressing newcomers' mental health issues ${ }^{7,19}$, it would provide an opportunity to further investigate some of the findings from the first study, and then to see if these findings would be replicated in New Zealand.

\section{Methods}

Focus groups, individual semi-structured interviews and a study questionnaire were used to collect data about participant resettlement experiences. Using the same cut off points as in the first study, the $\mathrm{DS}^{14}$ allowed for the degree of demoralisation among the participants in both samples to be determined. Interpreters were used throughout the studies.

\section{Sample}

In total 80 refugee and migrant people residents in Kitchener-Waterloo, Canada and who were attending one of two programmes (The Working Centre $=$ TWC, or the St Louise Learning Centre $=$ SLC) aimed at enhancing resettlement and 80 refugees and migrant people living in New Zealand and attending the Christchurch Resettlement Services (CRS) were involved in these two studies. Interpreters were used throughout the research process.

\section{Results}

It was found that in terms of mental health, $88 \%$ reported having been treated for depression in Canada and $81 \%$ in Christchurch. As shown in Table 2 below, the total DS mean score across all the agencies in studies two and three $($ mean $=25.34 ; \mathrm{SD}=14.47)$ indicated that while overall the DS total mean score would put participants at a mild level of demoralization, $(\mathrm{CRS}$ mean $=30.08 ; \mathrm{SD}=$ 15.01: TWC mean $=16.73 ; \mathrm{SD}=13.04$ and SLC mean $=23.32 ; \mathrm{SD}=11.06)$, the CRS participants had a higher, and trending toward a moderate, level of demoralization.

Generally, with the exception of being able to gain appropriate employment, the Canadian cohort findings indicated that the majority of the participants were able to speak Eng- 
Table 2

ANOVA showing significant mean effects of the items contained in the Demoralization scale between the TWC and SLC cohorts

\begin{tabular}{|c|c|c|c|c|}
\hline \multirow{3}{*}{ Offer value to others } & \multirow{2}{*}{$\begin{array}{l}\text { Mean (SE) } \\
\text { SLC }\end{array}$} & \multirow{2}{*}{$\frac{\text { F-ratio }}{1.43(0.13)}$} & \multicolumn{2}{|c|}{ P-value } \\
\hline & & & 6.027 & $0.016^{*}$ \\
\hline & TWC & $0.88(0.19)$ & & \\
\hline Emotionally & SLC & $1.17(0.16)$ & & \\
\hline uncontrolled & TWC & $0.64(0.16)$ & 5.238 & $0.025^{*}$ \\
\hline \multirow[t]{2}{*}{ I feel guilty } & SLC & $0.34(0.09)$ & & \\
\hline & TWC & $0.79(0.21)$ & 4.726 & $0.033^{*}$ \\
\hline \multirow[t]{2}{*}{ I cope well } & SLC & $1.70(0.18)$ & & \\
\hline & TWC & $1.09(0.23)$ & 4.536 & $0.036^{*}$ \\
\hline \multirow[t]{2}{*}{ Life is not worth living } & SLC & $0.62(0.15)$ & & \\
\hline & TWC & $0.06(0.04)$ & 9.183 & $0.003 * *$ \\
\hline \multirow[t]{2}{*}{ I am worthwhile } & SLC & $2.00(0.22)$ & & \\
\hline & TWC & $0.39(0.14)$ & 31.488 & $<0.001 * * *$ \\
\hline \multicolumn{5}{|l|}{$* * * \mathrm{p}<0.001$} \\
\hline \multicolumn{5}{|l|}{$* * \mathrm{p}<0.01$} \\
\hline$* \mathrm{p}<0.05$ & & & & \\
\hline
\end{tabular}

lish, felt safe, had a community identity and good social supports. The DS scores of the employed participants in Canada were slightly lower $($ mean $=19 ; \mathrm{SD}=11)$ than the unemployed participant DS scores $($ mean $=22$; SD13) however, all were still in the mild range.

In terms of achieving the NZSS goals in the Christchurch cohort less than half were proficient in English; very few (18\%) were employed; most felt safe in expressing their own identity; half felt accepted in the host community with two thirds reporting they had a community identity and good social networks. Thus, overall, the majority of the goals contained in the New Zealand Settlement Strategy were met, or partially met, with the exception of two key factors, having an ability to speak English and the lack of appropriate employment-both important factors for successful resettlement.
Lack of proficiency in English impacted significantly $(\mathrm{p}<0.01)$ on demoralization scores $($ mean $=32.48 ;$ SD $=14.15)$ in comparison to English speakers DS scores (mean $=21.20 ; \mathrm{SD}=12.32$ ). Unlike study one, no significant differences were found between the employed participants in the Christchurch cohort (DS mean score $=27$; SD 19.10) when compared with the unemployed (DS mean $=$ 31; SD.13.91).

The questionnaire used in studies two and three also contained a free text qualitative section where participants briefly recorded their negative and positive experiences of resettlement. Thematic coding was used for data analysis. In both cohorts, consistent with the quantitative findings, the dominant theme was the lack of appropriate employment, or having to accept positions that did not reflect their qualifications and training. 


\section{Discussion}

The literature details the complex relationship between the process of migration and the impact on an individual's development of psychological or psychiatric conditions. Successful resettlement does require considerable adaptation and if expectations are not met, an individual can become very distressed, disheartened and demoralized. This was evident in the first study where the clients admitted to a mental health service were more depressed and more demoralized than the participants attending the resettlement services. While this could be expected in people presenting to mental health services, it is worth noting that a significant association was also found between unemployment and their DS mean scores.

Although the Canadian cohort were less demoralized overall, a comparison across the clinical and non-clinical cohorts' in all three studies did find a degree of demoralization with an upward trend towards a moderate level. This adds support to a growing body of literature in relation to employment status. That is, gaining appropriate employment is a key factor $1,2,4,6,9,10,21,22$ in resettlement and can impact on the mental health well-being of an individual.

Furthermore, finding significant associations $(\mathrm{p}<0.01)$ between employment and demoralization scores reflects the benefits of employment - access to an income, resultant acceptable standard of living and greater acceptance in the host society. Denial of this opportunity can affect an individual's mental well-being ${ }^{1,4,22}$.

Additionally, gaining employment is often dependent on having an ability to communicate in the host country's major language $^{23}$ and being able to speak English, to the extent that an interpreter is not required, enhances an individual's ability to gain employment. While no significant associations were found between language and the DS in the first two studies, which could be expected given the high rate of English language ability in the two cohorts, this changed in the third study undertaken in Christchurch where significant associations between ability to speak English and the DS were found $(p<0.01)$.

It is pleasing to note that generally, with the exception of employment, the goals contained in the NZSS goals are achievable in Canada and that similarly in the main, in Christchurch. However, it is disconcerting to find that in all three studies gaining appropriate employment was a key issue and that in the third study, also proficiency in English. Both issues are written as goals in the NZSS which makes these important findings as there is significant research evidence that unemployment (especially long-term unemployment) has a negative effect on the wellbeing, settlement and/or adjustment process of immigrants and their families ${ }^{1,21,22}$.

As such, unemployment is associated not only with financial strain, but also with loss of self-esteem and restriction of social contact. It has functions other than providing income as it provides purpose to life, defines status and identity and enables individuals to establish relationships with others in the society. It is especially the latter function that becomes critical for refugees and migrants as adaptation is facilitated by social interactions. The more one interacts with the groups in the larger society, the faster one acquires skills to manage everyday life. Therefore, for those who are out of work, the result is not only a decline in psychological well-being, but also a delay in adaptation ${ }^{21,22}$. 


\section{Conclusion}

The findings from these studies do support the view that social and cultural issues play a role in understanding the degree of psychological distress among culturally diverse clients. Thus, in order to reduce the risk, additional factors associated with migration that may impact on the onset of mental health problems need to be taken into account. Indeed, demoralization does have much to offer in terms of enhancing intercultural understandings of psychological distress. However, it also raises the question as to whether the degree of demoralization found among refugee and migrant populations is a social-political problem or a psychological one. Evaluation of resettlement programmes and goals need to be undertaken with a commitment to integration, rather than assimilation, as psychological approaches only work with the problem and do not offer real solutions.

A social and political approach that will enable people to adjust more rapidly, to make full use of their skills and knowledge and that allows them to begin to restart their lives in ways that lead to a positive future is required.

\section{References}

1. Briggs L, Macleod AD (Sandy). Demoralization or clinical depression? Enhancing understandings of psychological distress in resettled refugees and migrants. World Cultural Psychiatry Research Review 2010; 5 (2): 86-98.

2. Nash M, Wong J, Trlin A. Civic and social integration: a new field of social work practice with immigrants, refugees and asylum seekers. Int Soc Work 2006; 49: 345-363.

3. Bhugra D. Migration and mental health. Acta Psychiatr Scand 2004; 109: 243-258.

4. Briggs L, Talbot C, Melvin K. An exploratory study of demoralization and migration experience. Int Rev Mod Sociol 2007; 33: 193-209.
5. Porter M, Haslam N. Predisplacement and postdisplacement factors associated with mental health of refugees and internally displaced persons: a meta-analysis. JAMA 2005; 294 (5): 602-612.

6. Beiser M, Hou F. Language acquisition, unemployment and depressive disorder among Southeast Asian refugees: a 10-year study. Soc Sci Med 2001; 53 (10): 1321-1334.

7. Canadian Task Force on Mental Health Issues Affecting Immigrants and Refugees. After the Door has Opened: Mental Health Issues Affecting Immigrants and Refugees. Ottawa: Minister of Supply and Services Canada; 1988.

8. Lavik NJ, Hauff E, Skrondal A, Solberg O. Mental disorder among refugees and the impact of persecution and exile: some findings from an out-patient population. Br J Psychiatry 1996; 169 (6): 726-732.

9. Pernice R, Brook J. Refugees' and Immigrants' mental health: Association of demographic and post-immigration factors. J Soc Psychol 1996; 136(4): 511-519.

10. Simich L, Hamilton H, Baya BK. Mental distress, economic hardship and expectations of life in Canada among Sudanese newcomers. Transcult Psychiatry 2006; 43 (3): 418-444.

11. Kissane DW, Clarke DM, Street AF. Demoralization syndrome -a relevant psychiatric diagnosis for palliative car. J Palliat Care 2001; 17: 12-21.

12. Clarke DM, Kissane DW. Demoralization: its phenomenology and Importance. Aust N Z J Psychiatry 2002; 36:733-742.

13. de Figueiredo JM. Depression and demoralization: phenomenological differences and research perspectives. Compr Psychiatry 1993; 34: 308-311.

14. Kissane DW, Wein S, Love A, Lee XQ, Kee PL, Clarke DM. The Demoralization Scale: a report of its development and preliminary validation. J Palliat Care 2004; 20: $269-276$

15. Beck AT, Steer RA, Brown GK. Beck Depression Inventory-II Manual. San Antonio (TX): The Psychological Corporation; 1996.

16. Beck AT, Weissman A, Lester D, Trexler L. The measurement of pessimism: the Beck Hopelessness Scale. J Consult Clin Psychol 1974 (4); 2: 861-865.

17. American Psychiatric Association. Diagnostic and Statistical Manual of Mental Disorders, $4^{\text {th }}$ edition (DSM-IV). Washington (DC): American Psychiatric Association; 1994.

18. Department of Labour Our Future Together: New Zealand Settlement Strategy. Wellington, New Zealand: New Zealand Government; 2007. 
19. Canadian Task Force on Mental Health Issues Affecting Immigrants and Refugees: Review of the Literature on Migrant Mental Health.Ottawa: Canadian Ministry of Supply and Services; 1988.

20. Clarke DM, Kissane DW, Trauer T, Smith GC. Demoralization, anhedonia and grief in patients with severe physical illness. World Psychiatry 2005, 4(2): 96-105.

21. Pernice R, Trlin A, Henderson A, North N, Skinner M. Employment status, duration of residence and mental health among skilled migrants to New Zealand: results of a longitudinal study. Int J Soc Psychiatry 2009; 55: 272-287.

22. Aycan Z, Berry JW. Impact of employment-related experiences on immigrants' psychological well-being and adaptation to Canada. Can J Behav Sci 1996; 28(3): 240-251.
Corresponding author:

Dr. Lynne Briggs, Associate Professor School of Human Services and Social Work, Griffith University

Gold Coast Campus, Parklands Drive, Southport QLD 4222, Australia

Email: 1.briggs@griffith.edu.au

Phone 0061755527466

Member: Aotearoa Association of Social Workers (ANZASW)

New Zealand Registered Social Worker 